\title{
Interfer(on)-ing with vascular repair after acute brain injury
}

Type I interferon response to systemic infections after head trauma or stroke impairs angiogenesis in injured tissues and may contribute to secondary neurological injury.

\section{Jeremy R. Herrmann and Dennis W. Simon}

$\mathrm{E}$ very year, millions of individuals worldwide suffer traumatic brain injury (TBI) or stroke, which are leading causes of acute brain injury-related morbidity and mortality ${ }^{1,2}$. A carefully orchestrated immune response to acute brain injury is crucial to promote repair and regeneration; however, a dysregulated immune response may contribute to secondary neuronal death and chronic neurodegeneration ${ }^{3}$. In this issue of Nature Immunology, Mastorakos and colleagues explore the introduction of a systemic infection after acute brain injury on the immune response and vascular repair in mouse models of TBI and hemorrhagic stroke ${ }^{4}$. This underexplored secondary insult is particularly relevant clinically, as patients hospitalized with TBI and stroke are at high risk of acquiring infection in the lung, urinary tract and bloodstream ${ }^{5}$.

Mastorakos and colleagues use a model of mild TBI (mTBI) in adult mice that was previously established by their group to investigate meningeal vascular injury ${ }^{6}$. Meningeal vascular disruption may be visualized in patients with mTBI using contrast magnetic resonance imaging and typically resolves within 2-3 weeks, although it may persist for months in a subset of patients. The model, in which the skull overlying the primary somatosensory cortex is unilaterally thinned and then manually compressed without fracture to induce injury to the underlying meninges, glial limitans and superficial neocortex, is characterized by meningeal vascular damage that is revascularized over a 7-day period in otherwise healthy mice.

The authors examined the effect of introducing systemic infection 4 days after injury using several representative paradigms in mice ${ }^{4}$. First, mice were infected intravenously with lymphocytic choriomeningitis virus (LCMV), which resulted in only $23 \%$ revascularization of the meningeal lesion by day 7 (Fig. 1). Notably, mice chronically infected with LCMV from birth (carriers) had normal vascular

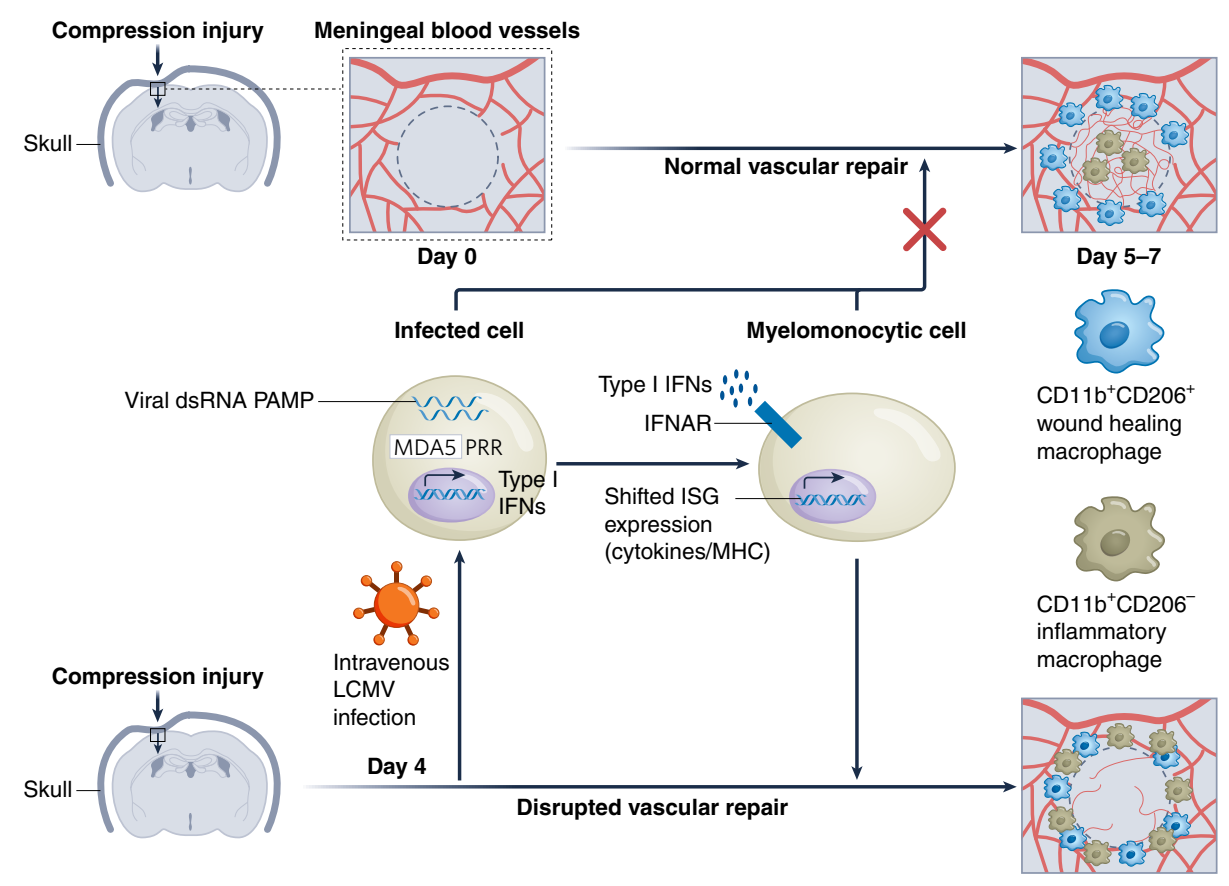

Fig. 1 | LCMV infection impairs vascular repair after TBI. Mice infected intravenously with LCMV on day 4 after a compression model of TBI demonstrate impaired vascular repair on day 7 as well as

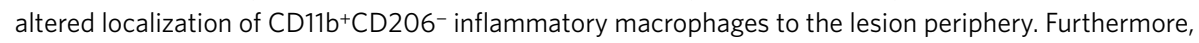
IFN-I signaling via IFNAR on myelomonocytic cells in response to LCMV infection shifts ISG expression and impairs vascular repair. Inhibition of viral recognition via the MDA5 pattern recognition receptor (PRR) or inhibition of IFN-I signaling restores vascular repair. dsRNA, double-stranded RNA; PAMP, pathogen-associated molecular pattern.

repair, which reveals the importance of the acute inflammatory response to infection in disrupting angiogenesis. Similar, but less robust, inhibition of vascular repair was seen with other infectious agents and pathogen-associated molecular patterns including intranasal vesicular stomatitis virus, systemic administration of polyinosinic:polycytidylic acid, lipopolysaccharide and Candida albicans. Mice infected with LCMV on day 4 after mTBI and then secondarily infected with vesicular stomatitis virus on day 10 developed chronic vascular disruption with only $50 \%$ repair at day 30 .
Having shown the time course of meningeal vascular repair after mTBI with and without infection, Mastorakos et al. ${ }^{4}$ then explored possible mechanisms for this finding. Given the breadth of infectious exposures that resulted in impaired healing in their model, and the lack of impairment in LCMV-carrier mice, a common immunological pathway was considered. The authors have previously reported a key temporal and spatial relationship of infiltrating macrophages with meningeal wound repair - inflammatory $\mathrm{CD}_{11 \mathrm{~b}^{+} \mathrm{CD} 206^{-} \text {cells in the lesion core }}$ and wound healing $\mathrm{CD} 11 \mathrm{~b}^{+} \mathrm{CD} 206^{+}$cells 
around the periphery of the lesion ${ }^{6}$. In their current work, Mastorakos et al. ${ }^{4}$ report that $\mathrm{CD} 11 \mathrm{~b}^{+} \mathrm{CD} 206^{-}$and $\mathrm{CD} 11 \mathrm{~b}^{+} \mathrm{CD} 206^{+}$ were both localized to the periphery of the lesion in LCMV-infected mice after TBI. The lack of clearance of cellular and extracellular debris from the lesion core by

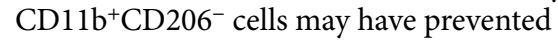
efficient revascularization.

LCMV has been used extensively to study type I interferons (IFN-I), which were the first endogenous anti-angiogenic factors identified by Brouty-Boye and Zetter in $1980^{7}$. Mastorakos et al. ${ }^{4}$ observed a marked increase in the expression of IFN-I in the meninges and pericontusional cortex in LCMV-infected mTBI mice relative to healthy controls, mTBI alone or LCMV infection alone, as well as a shift in the expression patterns of interferon-stimulated genes (ISGs). Genetic (Ifnar ${ }^{-/-}$and $M \mathrm{Ma5}^{-/-}$) or antibody-mediated inhibition of IFN-I signaling restored meningeal vascular repair in LCMV-infected mTBI mice. By contrast, transcranial or intravenous administration of IFN- $\beta$ alone blocked vascular repair in mTBI mice, although to a lesser extent than systemic LCMV infection. Mastorakos et al. ${ }^{4}$ further showed that genetically modified mice that lacked the IFN-I receptor $\left(\right.$ Ifnar $\left.^{-/}\right)$solely on myelomonocytic cells were not affected by LCMV infection, which suggests that IFN-I impairs vascular repair after TBI via myelomonocyte signaling.

To generalize their findings to an additional form of acute brain injury with vascular disruption, as well as to permit investigations of secondary neurological injury such as neuronal density and behavioral outcomes, which are difficult to assess in their mTBI model, Mastorakos et al. ${ }^{4}$ transitioned their studies from TBI to a hemorrhagic stroke model ${ }^{4}$. Using targeted pulsed ultrasound with intravenous injection of microbubbles to induce vascular injury $^{8}$, the authors again showed that LCMV-infection reduced vascular repair more than 25-fold in this model and associated with persistent permeability of the blood-brain barrier - which was restored in IFNAR- and MDA5-deficient mice. In hemorrhagic stroke, however, targeted deletion of IFNAR on myelomonocytes alone did not lead to full recovery of vascular repair, which suggests involvement of a distinct cell type in this injury model. Notably, in the hemorrhagic stroke model, Mastorakos et al. ${ }^{4}$ found reduced neuronal density in LCMV-infected mice and impaired spatial-reference memory in the Y-maze test relative to uninfected controls, which supports the conclusion that impaired vascular recovery due to systemic infection may negatively affect neurological outcome ${ }^{4}$.

These data provide compelling evidence that the immune response to systemic infection disrupts vascular repair after acute brain injury via IFN-I signaling. However, caution should be taken before extending the findings to a broader TBI or stroke population. For example, the spectrum of brain injuries after trauma is wide, ranging from contusion to penetrating injuries, and translating findings in one model to others within the TBI realm is a challenge with which the field continues to struggle. A multi-center consortium with expertise in several TBI models was established to improve the translation of pre-clinical findings and bring the most successful therapies into clinical trials ${ }^{9}$. Studies using the controlled cortical impact model of TBI, which is the most well-established and widely used pre-clinical model of TBI, have reported that genetic and antibody-mediated inhibition of IFN-I signaling is protective ${ }^{10}$. By contrast, investigations in spinal cord trauma have primarily found that administration of IFN- $\beta$ may be protective ${ }^{10}$. Investigations of epithelial wound healing also support a beneficial role for IFN-I via myeloid-epithelial cell interactions ${ }^{11,12}$.

Thus, the type of cellular damage, location of injury and time from injury may all need to be considered when predicting the effect of IFN-I on recovery from acute brain injury. However, although IFN-I signaling occurs as a component of the sterile inflammatory response to acute brain injury, the findings of Mastorakos et al. ${ }^{4}$ suggest that the degree associated with response to systemic infection may be universally detrimental to the host.

Ultimately, translating these findings into clinical practice may be challenging for neurointensivists and other clinicians caring for patients with acute brain injury. Although Mastorakos et al. ${ }^{4}$ demonstrate impaired meningeal vascular repair after administration of lipopolysaccharide and Candida in their TBI model, which are common infections that complicate the course of patients with TBI, the incidence of RNA viral infection after TBI or stroke, the focus of the mechanistic portions of this study, is unknown. Unfortunately, as the ongoing COVID-19 pandemic has demonstrated, treatment options for RNA viral infection in humans are limited. Although immunomodulatory treatments have failed to show benefit in patients with TBI or stroke ${ }^{3}$, and in some cases caused harm, patients with acquired infection after brain injury may benefit from immunomodulatory therapies in contrast to the broader populations of patients with TBI and stroke. Preventative measures such as vaccination and infection prevention protocols to limit nosocomial or household exposures to viral pathogens may be effective and should be investigated. Patients with acute brain injury may develop systemic immune suppression, and this study adds support to taking additional measures to prevent infection, although previous attempts to prevent acquired bacterial infection with empiric use of a broad-spectrum antibiotic after stroke showed no benefit in functional outcomes.

Mastorakos et al. ${ }^{4}$ have provided an important insight into the pathophysiology of vascular repair after acute brain injury. Instead of focusing on the injury mechanism itself, the group reveals the importance of secondary infections as an immune-modulating event that triggers faulty tissue repair and impairs functional outcomes. Future studies should attempt to validate these findings in other TBI and stroke models and identify new therapies and preventative measures that may be used to maintain or restore a beneficial immune response.

Jeremy R. Herrmann $n^{1,2}$ and

Dennis W. Simon (D) 1,2,3凶

${ }^{1}$ Department of Critical Care Medicine, University of Pittsburgh School of Medicine, Pittsburgh, PA, USA. ${ }^{2}$ Safar Center for Resuscitation Research, University of Pittsburgh School of Medicine, Pittsburgh, PA, USA. ${ }^{3}$ UPMC Children's Hospital of Pittsburgh Neuroscience Institute, University of Pittsburgh School of Medicine, Pittsburgh, PA, USA.

$\bigotimes_{e-m a i l}$ dennis.simon2@chp.edu

Published online: 23 September 2021 https://doi.org/10.1038/s41590-021-01034-9

References

1. GBD 2016 Traumatic Brain Injury and Spinal Cord Injury Collaborators. Lancet Neurol. 18, 56-87 (2019).

2. GBD 2016 Lifetime Risk of Stroke Collaborators. et al. N. Engl. J. Med. 379, 2429-2437 (2018).

3. Simon, D. W. et al. Nat. Rev. Neurol. 13, 171-191 (2017).

4. Mastorakos, P., Russo, M., Zhou, T., Johnson, K. \& McGavern, D. Nat. Immunol. https://doi.org/10.1038/s41590-021-01012-1 (2021).

5. Boehme, A. K. et al. Stroke 49, 2999-3005 (2018).

6. Russo, M. V., Latour, L. L. \& McGavern, D. B. Nat. Immunol. 19, 442-452 (2018).

7. Brouty-Boyé, D. \& Zetter, B. R. Science 208, 516-518 (1980).

8. Mastorakos, P. et al. Nat. Neurosci. 24, 245-258 (2021).

9. Kochanek, P. M. et al. J. Neurotrauma 33, 513-522 (2016).

10. Roselli, F., Chandrasekar, A. \& Morganti-Kossmann, M. C. Front. Neurol. 9, 458 (2018).

11. Di Domizio, J. et al. Nat. Immunol. 21, 1034-1045 (2020).

12. Sun, L. et al. Cell Host Microbe 17, 85-97 (2015).

Acknowledgements

This work was supported by the National Institutes

of Health: grant nos. T32HD040686 (J.R.H.) and

R21NS115174 (D.W.S.).

Competing interests

The authors declare no competing interests. 